\title{
Tennis Elbow- Mini Review
}

\author{
Arnav Wadhawan ${ }^{1 *}$, Harasees Singh ${ }^{2}$ and Amardeep Singh ${ }^{3 *}$ \\ ${ }^{1}$ California Northstate University, Rancho Cordova, USA \\ ${ }^{2}$ McMaster university, Hamilton, Canada \\ ${ }^{3}$ McMaster children hospital, Hamilton, Canada
}

Submission: April 06, 2021; Published: April 16, 2021

*Corresponding author: Arnav Wadhawan, California Northstate University, Rancho Cordova, USA,

Amardeep Singh, McMaster children hospital, Hamilton, Canada

\begin{abstract}
Lateral Epicondylitis, often referred to as tennis elbow, is a type of tendonitis affecting the common attachment of the tendons of the arm's extensor muscles to the lateral epicondyle of the humerus. It affects between 1-3\% of the population with most of the affected ranging between the ages of 30-35 years old. Lateral epicondylitis induces pain in the elbow during wrist and phalange extension, the onset of pain is gradual and can become chronic in more severe cases. Three main factors have been identified as part of the pathophysiology of lateral epicondylitis including, local tendon pathology, changes in the pain system and motor system impairment. These factors allow four stages to be classified with the first being able to be resolved without medical intervention, the fourth class containing soft matrix calcification and neovascularization. There is no literature that suggests one treatment modality that is the best in treatment for lateral epicondylitis however, the effectiveness of different treatments has been shown. One of the most straightforward strategies is watchful waiting in which the patient reduces any aggravating activity and completes regular follow-ups with their doctor. Other more involved treatment modalities are counterforce orthosis, corticosteroid injection and physical therapy. Counterforce orthosis has shown contradictory results in treatment, corticosteroid injections are very useful in shortterm pain relief with physical therapy providing more long-term benefits. However, these treatment modalities have marginal improvements compared to watchful waiting, as well the different types of surgery can aid in treatment of more severe cases.
\end{abstract}

Keywords: Tennis elbow; Lateral epicondylitis; Wrist dorsiflexion; Hyperalgesia

\section{Introduction}

Lateral epicondylitis, referred to as tennis elbow, was first described in medical literature by Runge in the late 1800s [1]. It was initially described as an inflammatory condition. However, it has since been reclassified as tendinosis, which is a term used to describe a chronic symptomatic degeneration of the tendons. In the case of lateral epicondylitis, the tendinosis affects the common attachment of the tendons of the arm's extensor muscles to the lateral epicondyle of the humerus. These muscles include the extensor carpi radialis brevis, the extensor carpi radialis longus, the extensor carpi ulnar is, extensor digitorum, and the extensor digiti minimi [2]. The main population of people affected by this injury is between 33-55 years; there is no change in incidence rate through gender [1]. Even though it affects $1-3 \%$ of the population, there is no universal treatment [3]. Many cases are self-limiting and are managed with pain medication, with most recovering in one year. However, chronic or severe lateral epicondylitis presentations can be treated by a variety of treatment modalities with conservative and operative options available.

\section{Anatomy}

Lateral epicondylitis results in pain in the elbow during wrist and phalange extension, as tendinosis occurs due to an injury of the common extensor tendon. Mobility during extension movements causes pain when the extensor muscles extensor carpi radialis brevis, the extensor carpi radialis longus, the extensor carpi ulnaris, extensor digitorum, and the extensor digiti minimi are used. In the extensor muscles, the extensor carpi radialis brevis is the most commonly affected during lateral epicondylitis [4].

\section{Signs and Symptoms}

The most pertinent symptom of lateral epicondylitis is a sharp, intermittent pain in the region of the distal lateral humerus that radiates on the arm's extensor surface. This pain usually has a gradual onset, although the pain may become acute after partaking in activities that involve the pronation of the arm when the elbow is fully extended. These activities can range from elite level tennis to turning a doorknob [5]. In more severe cases, there can 


\section{Orthopedics and Rheumatology Open Access Journal (OROAJ)}

be constant and extreme pain which disturbs daily activities or sleep. Physical examination of the elbow during diagnosis is only used for differential diagnosis to rule out any other possible conditions that may cause this type of pain [5]. The examination does not reveal abnormalities except in patients with a long-standing disease where the partial or complete rupture of the common extensor tendon is present or in patients who have used corticoid steroids as the overlying skin may be depigmented or thin. Palpation of the insertion of the extensor carpi radialis brevis tendon showing tenderness may also indicate lateral epicondylitis. During the examination, absence of trigger points must also be ascertained to rule out other conditions. This leads to the physical exam being more useful to ensure the pain is not a result of a more complicated condition.

\section{Susceptibility and Prevention}

In an observational study with 5800 participants, only $1.3 \%$ developed lateral epicondylitis [6]. In this study, the risk factors ranged from labor workers who complete repetitive forceful activities of handling heavy loads of more than $20 \mathrm{~kg}$ being between ages 45 to 55 to the lesser thought of environmental factors such as smoking and obesity. Labor workers are likely to get this injury in their dominant side during high physical strain. Another key risk factor is if the symptoms such as pain last for more than three months. Therefore, limiting the strenuous activity in labor positions may decrease the risk of lateral epicondylitis. Tennis players are at a higher chance of this injury as elbow injuries account for $20 \%$ of all tennis-related injuries. In a study of 529 players, lateral epicondylitis occurred more frequently in older players with a $50 \%$ risk in players over the age of 30 with increased risk in players over 40 [6]. It was also reported that players who played more than two hours a day had a much higher chance of injury [6]. In another study of 700 players in a professional tournament over three years, it was found that only one incidence of lateral epicondylitis occurred, leading to the finding that this injury is more common in recreational players due to poor technique [6]. Therefore, in tennis players, the proper technique may go a long way in reducing this injury.

\section{Aetiology}

There is not much research on how lateral epicondylitis happens as it impacts $1 \%$ to $3 \%$ of adults each year. It was thought initially that lateral epicondylitis occurred from continued intense activity while playing tennis [7]. However, it has since been reported that only $5 \%-10 \%$ of patients with this injury played tennis, [7] and is more common in people who do not play sports. It is now understood to occur due to repetitive and strenuous activity during wrist dorsiflexion with supination or pronation. Wrist dorsiflexion is used among various activities such as typing, explosive movements by athletes, or regular handling of loads over $20 \mathrm{Kg}$ [6]. These activities lead to microlesions at the extensor tendon, which starts the injury's degenerative aspect, leading to angiofibroblastic proliferation [7].

\section{Pathophysiology}

Three main factors have been identified as part of the pathophysiology of lateral epicondylitis. These include local tendon pathology, changes in the pain system, and motor system impairment [8]. The combination of these factors over time allows for four main stages of the injury to be classified:

a) Stage 1: an acute inflammatory response that may resolve without medical intervention. In further stages, there is an absence of inflammatory cells suggesting lateral epicondylitis is not an inflammatory process [9].

b) Stage 2: If stage one is not resolved independently, angiofibroblastic hyperplasia can occur, including an increase in ground substance and cell number, vascular hyperplasia, and disorganized and immature collagen. Hypercellularity can occur in both organized and disorganized muscle fiber orientation. This is the stage at which presentation for treatment is most common. [1].

c) Stage 3: Further progression of this disease leads to tendinosis or tendon tearing. Ultrasonography has demonstrated this through the prevalence of changing tendon size and tendon tears during this stage [10].

d) Stage 4: In the final and most severe stage of this disease, fibrosis, tendon tears, and soft matrix calcifications are present. Doppler ultrasonography has even shown neovascularization during this stage [10].

During the progression of lateral epicondylitis, pain perception may also be changed. This is because lateral epicondylitis is associated with hyperalgesia as well as an increased response to stimuli. The hyperalgesia can also spread to other sites, including over the cervical spine [11]. The decreased pain threshold may be due to pain modulator substances in the extensor carpi radials brevis tendon. Evidence suggests that these pain modulator substances contribute to lateral epicondylitis progression as they stimulate proliferation and matrix synthesis of fibroblasts [12].

Due to the progression of lateral epicondylitis, motor system impairment may occur, leading to diminished strength due to morphological differences in the muscle [8]. The maximum handgrip and pain-free handgrip strength decreased with specific strength deficits occurring in the wrist, hand, and shoulder. The reasons for these deficits can be seen through the histological examination of the extensor carpi radialis brevis. There are many muscle cells changes in this muscle, such as fiber necrosis, signs of muscle fiber regeneration, and an increased proportion of type 2A fast-twitch oxidative muscle fibers [13].

\section{Treatment}

Currently, there is no literature on the topic to suggest that one type of treatment for lateral epicondylitis is better [14]. However, meta-analyses and randomized controlled trials have shown the effectiveness of varying treatment options such as watchful waiting, medications, physical therapy, and surgery. 
The most straightforward lateral epicondylitis injuries can be completely healed by watchful waiting. In this case, the patient spends one year without aggravating activities to the elbow and frequently follows-up with their doctor [14]. During this period, ergonomic instructions are given with prescriptions of heat, cold, braces, and analgesic drugs [15]. Another simple form of treatment comes from counterforce orthosis. In this type of lateral epicondylitis management, an orthotic device is used to alleviate pain and reduce inflammation. However, these devices have little to no effect on increasing strength or proprioception [16]. Another study concluded that due to orthotic devices' ability to reduce pain, they should be used during the initial phases of treatment [17]. Many studies have been conducted comparing the effects of different types of orthotic devices. However, the findings are contradictory [18]. This leads to the suggestion that more research be conducted on orthotic device use in the treatment of lateral epicondylitis.

Another type of treatment is corticosteroid injection. This treatment modality has been shown to provide more significant short-term pain relief and improved grip strength than other modalities. However, after a few months, the opposite was found, with corticosteroids providing the least benefit in all outcome measures compared to the wait and see policy and physiotherapy. As well, studies found no significant difference in the type of corticosteroid injection used [19]. The reason for the decreased effects of corticosteroids over long periods is currently unknown. However, it has been hypothesized that corticosteroid injections might be harmful to the tendon and that the rapid improvement of pain may lead to increased activity of the tendon and overtaxing [15].

Physical therapy has been shown to provide more substantial and more effective benefits in the long run. This modality uses exercise and nonsteroidal anti-inflammatory drugs [14]. The nonsteroidal anti-inflammatory drugs are used for the first four weeks to provide short-term pain relief. For long-term pain relief, stretching and strengthening exercises are used. Exercise programs have been shown to reduce pain, but improvement in grip strength is still unclear. It is also essential to focus on eccentric phases of exercise as they provide the most benefit.

The main concern of physical therapy is cost-effectiveness. As physical therapy has marginally better results when compared to watchful waiting [20]. However, physical therapy requires at least one session with a physical therapist, leading to much higher costs than corticosteroid injections or watchful waiting. [15]. While these injuries can be healed over time, more complex injuries may need surgical intervention. This method is only recommended if other conservative methods do not improve the symptoms after six to twelve months [14]. Surgery is an encompassing method with different options that range from removing the abnormal tissue to releasing the tendon itself. Some case studies have suggested potential benefits, yet more evidence is needed for a conclusion to be made.

\section{Conclusion}

Due to the limited evidence comparing the different types of treatments for lateral epicondylitis it is not possible to suggest one treatment for all cases. The most common treatment modality is watchful waiting with follow-ups with a doctor. However, more severe cases can benefit from more active measures such as physical therapy and corticosteroid injections. However, both methods have their caveats. More research is needed to suggest the use of orthotic devices or surgery.

\section{References}

1. Ahmad Z, Siddiqui N, Malik SS, Abdus SM, Tytherleigh SG, et al. (2013) Lateral epicondylitis: a review of pathology and management. Bone Joint J 95-B(9): 1158-1164.

2. Mitchell B, Whited LA (2020) Shoulder and Upper Limb, Forearm Muscles. In: StatPearls [Internet]. Treasure Island (FL): Stat Pearls Publishing, FL, USA.

3. Keijsers R, De Vos RJ, Kuijer PPF, Van Den Bekerom MP, Van Der Woude HJ, et al. (2019) Tennis elbow. Shoulder Elbow 11(5): 384-392.

4. Cyriax JH (1936) The Pathology and Treatment of Tennis Elbow. The Journal of Bone and Joint Surgery 18(4): 921-940.

5. Geoffroy P, Yaffe MJ, Rohan I (1994) Diagnosing and treating lateral epicondylitis. Can Fam Physician 40: 73-78.

6. Jayanthi N, Fields K, Grayzel J (2018) Elbow tendinopathy (tennis and golf elbow).

7. Cohen M, Da Rocha MFG (2015) Lateral Epicondylitis of the Elbow. Rev Bras Ortop 47(4): 414-420.

8. Coombes BK, Bisset L, Vicenzino B (2009) A new integrative model of lateral epicondylalgia. Br J Sports Med 43(4): 252-258.

9. Luk JKH, Tsang RCC, Leung HB (2014) Lateral epicondylalgia: midlife crisis of a tendon. Hong Kong Med J 20(2): 145-151.

10. Connell D, Burke F, Coombes P, McNealy S, Freeman D, et al. (2001) Sonographic Examination of Lateral Epicondylitis. AJR Am J Roentgenol 176(3): 777-782.

11. Berglund KM, Persson BH, Denison E (2008) Prevalence of pain and dysfunction in the cervical and thoracic spine in persons with and without lateral elbow pain. Man Ther 13(4): 295-299.

12. Uchio Y, Ochi M, Ryoke K, Sakai Y, Ito Y, et al. (2002) Expression of neuropeptides and cytokines at the extensor carpi radialis brevis muscle origin. J Shoulder Elbow Surg 11(6): 570-575.

13. Ljung BO, Lieber RL, Fridén J (1999) Wrist extensor muscle pathology in lateral epicondylitis. J Hand Surg Br 24(2): 177-183.

14. Johnson GW, Cadwallader K, Scheffel SB, Epperly TD (2007) Treatment of lateral epicondylitis. Am Fam Physician 76(6): 843-848.

15. Smidt N, Van der Windt DA (2006) Tennis elbow in primary care. BMJ 333(7575): 927-928.

16. Ng GYF, Chan HL (2004) The Immediate Effects of Tension of Counterforce Forearm Brace on Neuromuscular Performance of Wrist Extensor Muscles in Subjects with Lateral Humeral Epicondylosis. J Orthop Sports Phys Ther 34(2): 72-78.

17. Sadeghi DE, Jafarian F (2013) The Immediate Effects of Orthoses on Pain in People with Lateral Epicondylalgia. Pain Research and Treatment p.1-6. 
18. Vellilappilly DV, Rai HR, Varghese J, Renjith V (2017) Counterforce Orthosis in the Management of Lateral Epicondylitis. J Ayub Med Coll Abbottabad 29(2): 328-334.

19. Hsieh L, Kuo Y, Lee C, Liu Y, Liu Y, et al. (2018) Comparison Between Corticosteroid and Lidocaine Injection in the Treatment of Tennis Elbow. American Journal of Physical Medicine \& Rehabilitation 97(2): 83-89.
20. Bisset L, Beller E, Jull G, Brooks P, Darnell R, et al. (2006) Mobilisation with movement and exercise, corticosteroid injection, or wait and see for tennis elbow: randomised trial. BMJ 333(7575): 939-941.

\section{Your next submission with Juniper Publishers} will reach you the below assets

- Quality Editorial service

- Swift Peer Review

- Reprints availability

- E-prints Service

- Manuscript Podcast for convenient understanding

- Global attainment for your research

- Manuscript accessibility in different formats ( Pdf, E-pub, Full Text, Audio)

- Unceasing customer service

Track the below URL for one-step submission https://juniperpublishers.com/online-submission.php 